\title{
Electronic polarons in an extended Falicov-Kimball model
}

\author{
P. M. R. Brydon ${ }^{1}$, M. Gulácsi ${ }^{1}$ and A. R. Bishop ${ }^{2}$ \\ 1 Department of Theoretical Physics, Institute of Advanced Studies, \\ The Australian National University, Canberra, ACT 0200, Australia \\ 2 Theoretical Division, Los Alamos National Laboratory, Los Alamos, NM 87545, U.S.A.
}

(Dated: November 10, 2018)

\begin{abstract}
We examine the one-dimensional spinless Falicov-Kimball model extended by a hybridization potential between the localized and itinerant electron states. Below half-filling we find a crossover from a mixed-valence metal to an integer-valence phase separated state with increasing on-site Coulomb repulsion. This crossover regime is characterized by local competition between the strongand weak-coupling behaviour, manifested by the formation of an electronic polaron liquid. We identify this intermediate-coupling regime as a charge-analogy of the Griffiths phase; a phase diagram is presented and discussed in detail.

PACS numbers: 71.28.+d, 71.27.+a
\end{abstract}


The Falicov-Kimball model (FKM) has found widespread use as a model of the charge physics in mixed-valence (MV) materials. Although the FKM's description of a band of itinerant conduction (c-)electrons interacting via a repulsive contact potential $G$ with an underlying lattice of localized $(f$-)electrons retains its relevance, $\stackrel{1}{=}$ the great evolution of our understanding of MV systems has required modification of the model's original form. $\underline{\underline{2}}^{2}$ The most popular such modification is the assumption of some overlap between the $c$ - and $f$-wavefunctions: this yields the extended or quantum Falicov-Kimball model (QFKM).

The ground state of the QFKM has for many years been a matter of controversy, with two contradictory pictures common in the literature. The homogeneous solution $\underline{\underline{3}}$ assumes a simple renormalization of the $G=0$ band structure by the Coulomb interaction; at meanfield level, this has been used to deduce the existence of a novel electronic ferroelectric MV state. $\stackrel{\underline{4}}{ }$ On the other hand, the relationship of the single-impurity QFKM to the Kondo impurity ${ }^{\underline{5}}$ suggests distinct strong- and weak-coupling regimes in the lattice model..$^{6.7}$

The latter scenario leads to interesting properties of the intermediate-coupling regime, specifically the formation of electronic polarons $\underline{\underline{7.8}}$ This electronic polaron model has recently been used to explain the transport properties of certain Heusler and Ce alloys; ${ }^{\frac{9}{}}$ more intriguingly, such polaronic structures play a pivotal role in the theory of the exotic "valencefluctuating" superconductivity in $\mathrm{CeCu}_{2} \mathrm{Ge}_{2}$ and $\mathrm{CeCu}_{2} \mathrm{Si}_{2} \cdot \stackrel{10}{\underline{10}}$ It is therefore of considerable interest to study the formation of electronic polarons in the QFKM.

In this letter we address this question by a non-perturbative study of the one-dimensional (1D) QFKM below half-filling. We derive an effective Hamiltonian for the occupation of the $f$-orbitals which gives a complete description of the physics. This allows us to exactly study the intermediate-coupling regime, where we find local competition between the strong- and weak-coupling phases. This produces a charge analogy of the Griffiths phase, which may be visualized as a gas of electronic polarons moving in a MV background. A schematic phase diagram is presented and discussed in detail.

The 1D QFKM for spinless fermions has the Hamiltonian

$$
\mathcal{H}_{\mathrm{QFKM}}=-t \sum_{\langle i, j\rangle} c_{i}^{\dagger} c_{j}+\epsilon_{f} \sum_{j} n_{j}^{f}+G \sum_{j} n_{j}^{c} n_{j}^{f}+V \sum_{j}\left\{c_{j}^{\dagger} f_{j}+\text { H.c. }\right\}
$$

Some overlap between the $c$ - and $f$-electron wavefunctions is assumed hence the on-site hybridization term $V \ll t$. The concentration of electrons is fixed at $n=(1 / N) \sum_{j}\left\{n_{j}^{f}+n_{j}^{c}\right\}$ where $N$ is the number of sites. In general we have $n<1$ due to the presence of doped 
impurities. We assume a MV state at $G=0$, requiring the Fermi level to be pinned at $-2 t<\epsilon_{f}<-2 t \cos (n \pi)$ in the noninteracting system. ${ }^{2}$

Bosonization is an asymptotically exact method, rigorously describing the longwavelength collective behaviour of the itinerant electrons, ${ }^{12}$ the short-range behaviour remains fermionic, however, and a cut-off $\alpha$ on the wavelength of the bosonic density fluctuations is therefore imposed. ${ }^{11}$ In simple cases such as the Hubbard model, $\alpha$ can be taken as vanishing. This is not true for systems such as the QFKM where the interactions with the localized orbitals determine the lattice constant as the minimum physical length scale. We are thus required to assume finite $\alpha>a$ : in the QFKM $\alpha$ parameterizes the electron delocalization length.

A finite wavelength cut-off may be easily included into the standard bosonization formalism. ${ }^{11}$ In particular, for the linearized fermionic fields about the Fermi points the well-known Mandelstram representation still holds

$$
c_{\nu j}=\sqrt{\frac{A a}{\alpha}} \hat{F}_{\nu} \exp \left(-i \nu\left[\phi\left(x_{j}\right)-\nu \theta\left(x_{j}\right)\right]\right)
$$

where $\phi$ and $\theta$ are the dual Bose fields, and the subscript $\nu=L(-), R(+)$ as subscript (otherwise) for the left- and right-moving fermions respectively. The Bose fields are defined in terms of particle-hole excitations about the two Fermi points. For a system of length $L \gg a$ we have

$$
\begin{aligned}
\phi\left(x_{j}\right) & =-i \sum_{\nu} \sum_{k \neq 0} \frac{\pi}{k L} \rho_{\nu}(k) \Lambda_{\alpha}(k) e^{i k x_{j}} \\
\theta\left(x_{j}\right) & =-i \sum_{\nu} \sum_{k \neq 0} \nu \frac{\pi}{k L} \rho_{\nu}(k) \Lambda_{\alpha}(k) e^{i k x_{j}}
\end{aligned}
$$

where $\rho_{\nu}(k)=\sum_{k^{\prime}} c_{\nu, k^{\prime}-k}^{\dagger} c_{\nu, k^{\prime}}$ are the particle-hole density operators. The wavelength cut-off is enforced in Eq. (3) and Eq. (41) by the function $\Lambda_{\alpha}(k)$ : this function satisfies the conditions $\Lambda_{\alpha}(k) \approx 1$ for $|k|<\alpha^{-1}$ and $\Lambda_{\alpha}(k) \approx 0$ otherwise. The numerical constant $A$ in Eq. (2) is determined by the functional form of $\Lambda_{\alpha}(k) . \hat{F}_{\nu}$ is the so-called Klein factor ${ }^{11.12}$

Eq. (2) correctly reproduces the long-wavelength $(\gg a)$ fermionic anticommutators and correlation functions. Since the Bose fields cannot resolve separations less than $\alpha$, however, the Mandelstram identity breaks down at short distances $\sim a$. This is reflected in the Bose field commutators, which are "smeared" by the cut-off function. For example, assuming exponential cut-off $\Lambda_{\alpha}(k)=\exp (-\alpha|k| / 2)$ we have $\left[\phi\left(x_{j}\right), \theta(0)\right]_{-}=-i \arctan \left(x_{j} / \alpha\right)$ and 
$\left[\partial_{x} \phi\left(x_{j}\right), \theta(0)\right]_{-}=-i \alpha\left[\alpha^{2}+\left(x_{j}\right)^{2}\right]^{-1}$. In the limit $\alpha \rightarrow 0$ we recover the Luttinger model forms of a sign and Dirac delta function respectively $\underline{\underline{12}}$

Substituting Eq. (2) into Eq. (11) and applying standard field-theory techniques, it is a simple matter to obtain the bosonized form of the QFKM Hamiltonian. For insight into the physics, however, we must rotate the Hilbert space to couple the $c$ - and $f$-electron orbitals. To accomplish this, we apply a lattice generalization of the shift transformation used by Schotte and Schotte in the X-ray edge problem, $\hat{U}=\exp \left\{i \frac{G a}{\pi v_{F}} \sum_{j}\left(n_{j}^{f}-\frac{1}{2}\right) \theta\left(x_{j}\right)\right\} \underline{\underline{13}}$ The Fermi velocity is defined $v_{F}=2 \operatorname{ta} \sin \left(k_{F} a\right)$ where $k_{F}=n_{0}^{c} \pi / a$, with $n_{0}^{c}$ the noninteracting $c$-electron concentration.

In the transformed Hamiltonian we combine the $f$-electron operators and the Klein factors into pseudospins using a generalized Jordan-Wigner transformation $\tau_{j}^{z}=n_{j}^{f}-\frac{1}{2}, \tau_{j}^{+}=$ $f_{j}^{\dagger} \hat{F}_{\nu} e^{-i \nu \pi x_{j} / 2 a} \exp \left(-i \frac{\nu \pi}{2} \sum_{j^{\prime}} \operatorname{sgn}\left(x_{j^{\prime}}-x_{j}\right)\left(n_{j^{\prime} \sigma}^{f}-\frac{1}{2}\right)\right)$. Note the relationship of $\tau_{j}^{z}$ to the occupation of the $f$-orbital at site $j$. We then write the transformed Hamiltonian in its final form

$$
\begin{aligned}
\hat{U}^{\dagger} \mathcal{H}_{\mathrm{FKM}} \hat{U}= & \frac{v_{F} a}{2 \pi} \sum_{j}\left\{\left(\partial_{x} \phi\left(x_{j}\right)\right)^{2}+\left(\partial_{x} \theta\left(x_{j}\right)\right)^{2}\right\}+G\left(n_{0}^{c}-\frac{1}{2}\right) \sum_{j} \tau_{j}^{z} \\
& -\frac{G^{2} a^{2}}{2 \pi^{2} v_{F}} \sum_{j, j^{\prime}} \tau_{j}^{z}\left\{i\left[\partial_{x} \phi\left(x_{j}\right), \theta\left(x_{j^{\prime}}\right)\right]_{-}\right\} \tau_{j^{\prime}}^{z} \\
& -\frac{2 G A a}{\alpha} \sum_{j} \tau_{j}^{z} \cos \left(2\left\{\phi\left(x_{j}\right)-\mathcal{K}_{\alpha}\left(x_{j}\right)-\left[k_{F}+\frac{\pi}{2 a}\right] x_{j}\right\}\right) \\
& +2 V \sqrt{\frac{A a}{\alpha}} \sum_{j}\left\{\tau_{j}^{+} e^{i\left(1-\frac{G a}{\pi v_{F}}\right) \theta\left(x_{j}\right)} \cos \left(\phi\left(x_{j}\right)-\mathcal{K}_{\alpha}\left(x_{j}\right)-\left[k_{F}+\frac{\pi}{2 a}\right] x_{j}\right)+\text { H. (.5 } 5\right)
\end{aligned}
$$

where $\mathcal{K}_{\alpha}\left(x_{j}\right)=\mathcal{S}_{\alpha}\left(x_{j}\right)+\mathcal{L}_{\alpha}\left(x_{j}\right), \mathcal{S}_{\alpha}\left(x_{j}\right)=\sum_{n=1}^{\infty}\left[\left\{i\left[\theta\left(x_{j+n}\right), \phi\left(x_{j}\right)\right]_{-}\right\}-\frac{\pi}{2}\right]\left(\tau_{j+n}^{z}-\tau_{j-n+1}^{z}\right)$ and $\mathcal{L}_{\alpha}\left(x_{j}\right)=\left(\frac{G a}{\pi v_{F}}-1\right) \sum_{n=1}^{\infty}\left\{i\left[\theta\left(x_{j+n}\right), \phi\left(x_{j}\right)\right]_{-}\right\}\left(\tau_{j+n}^{z}-\tau_{j-n}^{z}\right)$. We have kept all terms produced by the canonical transform in Eq. (5).

The three terms in Eq. (5) arising from the Coulomb interaction are of special note. The first term represents a renormalization of the $f$-level energy and drives a valence transition with increasing $G$. For $0 \leq n_{0}^{c}<0.5\left(0.5<n_{0}^{c} \leq 1\right)$ the negative (positive) sign of this energy-shift implies the emptying of the $c$-electron ( $f$-electron) band so that all electrons have an unambiguously $f$-electron (c-electron) character. The case $0 \leq n_{0}^{c}<0.5$ is particularly interesting as here the Ising interaction term orders the available $f$-electrons into a single contiguous block, the well-known segregated (SEG) phase of the $V=0$ limit $\underline{\underline{15}}$ The origin of this interaction is the finite spread $\alpha>a$ of the $c$-electron wavefunctions: to mini- 
mize the interorbital Coulomb repulsion this favours empty underlying $f$-orbitals. The last term originates from the backscattering of the $c$-electrons off the localized orbitals. In the absence of the hybridization, this will order the available $f$-electrons into crystalline phases via a Peierls-like mechanism $\frac{16}{\underline{6}}$ The addition of the hybridization, however, replaces these phases by a MV state; in the following analysis we therefore neglect the backscattering. Including this effect does not qualitatively alter our conclusions $\underline{\underline{17}}$

The phases of the QFKM are most usefully classified in terms of the occupation of the localized orbitals. We therefore derive an effective Hamiltonian only for the $f$-occupation (i.e. the $\tau$-pseudospins). This is straightforwardly achieved by replacing the Bose fields in Eq. (15) with suitably chosen expectation values. At weak-coupling, Schlottmann's renormalization group study reveals that the system flows to the $G=0$ fixed point; $\underline{\underline{6}}$ at strong-coupling, where the system is in the SEG phase, exact diagonalization calculations by Farkašovský in the $V=0$ limit indicate that the $c$-electrons are at their noninteracting fixed point $\underline{\underline{14}}$ On the basis of these studies it is natural to take $\left\langle\phi\left(x_{j}\right)\right\rangle=\left\langle\theta\left(x_{j}\right)\right\rangle=0$ across the phase diagram. We hence obtain the effective Hamiltonian

$$
\mathcal{H}_{\mathrm{eff}}=-\mathcal{J} \sum_{j} \tau_{j}^{z} \tau_{j+1}^{z}+G\left(n_{0}^{c}-\frac{1}{2}\right) \sum_{j} \tau_{j}^{z}+4 V \sqrt{\frac{A a}{\alpha}} \sum_{j} \tau_{j}^{x} \cos \left(\mathcal{K}_{\alpha}\left(x_{j}\right)+\left[k_{F}+\frac{\pi}{2 a}\right] x_{j}\right)
$$

We have approximated the segregating interaction by its nearest-neighbour form where $\mathcal{J}=$ $\frac{G^{2} a^{2}}{\pi v_{F}} \frac{1}{L} \sum_{k} \Lambda_{\alpha}(k) e^{i k a}$.

$\mathcal{H}_{\text {eff }}$ is instantly recognizable as a transverse field Ising model. For large $G$, we recover the SEG phase as the Ising term dominates the Hamiltonian. In the limit of vanishing Coulomb repulsion, however, the site-dependent transverse field $h_{j}^{x}=4 V \sqrt{\frac{A a}{\alpha}} \cos \left(\mathcal{K}_{\alpha}\left(x_{j}\right)+\right.$ $\left.\left[k_{F}+\frac{\pi}{2 a}\right] x_{j}\right)$ dominates Eq. (66). The pseudospins are therefore ordered along the $x$-axis with $\left\langle\tau_{j}^{x}\right\rangle \approx \frac{1}{2} \operatorname{sgn}\left(h_{j}^{x}\right)$. Translated back into the $f$-occupation 'language' this implies that $\left\langle f_{j}^{\dagger} c_{j}+\right.$ H.c. $\rangle \neq 0$, with the occupation of each $f$-site being given by $\left\langle n_{j}^{f}\right\rangle \approx n_{0}^{f}$ where $n_{0}^{f}$ is the $f$-electron concentration for $G=0$. This clearly corresponds to a MV state.

The MV and SEG phases are characterized by quite contradictory behaviours of the $f$ electrons: in the former the localized orbitals are in a superposition of their different valence states, while in the latter there is a phase separation between regions with different integer ionic valence. At coupling strengths intermediate between the MV and SEG phases, therefore, we expect to observe some competition between these two distinct charge physics. For a vanishing longitudinal field, Eq. (6) displays an order-disorder transition; the longitudinal 
field however lifts the system from criticality and we instead find a crossover between the ordered and disordered phases $\frac{18}{\underline{-}}$ To understand the details of the crossover regime, we must examine the site-dependence of $h_{j}^{x}$; specifically, we study the operator $\mathcal{K}_{\alpha}\left(x_{j}\right)$, which we replace by its expectation value.

The string operator $\mathcal{K}_{\alpha}\left(x_{j}\right)$ consists of two contributions, one measuring the short-range $\left[\mathcal{S}_{\alpha}\left(x_{j}\right)\right]$ and the other the long-range $\left[\mathcal{L}_{\alpha}\left(x_{j}\right)\right]$ ordering of the pseudospins. In the SEG phase, the short-range correlations of the pseudospins are very strong and so $\mathcal{S}_{\alpha}\left(x_{j}\right)$ vanishes; the importance of disorder in the crossover regime, however, means that here $\mathcal{S}_{\alpha}\left(x_{j}\right)$ has a random variation. In contrast, the long-range ordering of the pseudospins in the SEG phase persists deep into the crossover regime. $\mathcal{L}_{\alpha}\left(x_{j}\right)$ is only non-zero for $0 \leq n_{0}^{c}<0.5$ where it takes a maximum at the edge of the $f$-electron block, increasing linearly as this point is approached from either side: for the boundary of the $f$-block at $x_{0}$ we find $\mathcal{L}_{\alpha}\left(x_{j}\right) \sim\left(\frac{G a}{\pi v_{F}}-1\right)|j|$. The linear variation of $\mathcal{L}_{\alpha}\left(x_{j}\right)$ implies a quasiperidoic transverse field in the effective Hamiltonian Eq. (6). The periodicity of this field is in general incommensurate with the lattice: numerical ${ }^{19}$ and analytical ${ }^{20}$ studies have revealed the physics to be qualitatively identical to a random variation of transverse field. Fisher has used a real-space renormalization group method to exmaine in detail this random transverse field Ising model. $\underline{\underline{\underline{ }}}$ To utilise his detailed results we replace the transverse field in Eq. (6) by a field $\widetilde{h}_{j}^{x}$ which varies randomly with $j$, the values being drawn from a cosine distribution $\rho(\widetilde{h}) d \widetilde{h}=(C \pi)^{-1} \sqrt{1-(\widetilde{h} / C)^{2}}$ where $C=4 V \sqrt{A a / \alpha}$.

Following Fisher we find it convenient to define the dimensionless parameter $\delta=$ $\log \left(G_{c} / G\right)$ where $G_{c}^{2}=k t \sin \left(n_{0}^{c} \pi\right) V$. Assuming exponential cut-off, the proportionality constant takes the form $k=8 \sqrt{\frac{2 \pi a}{\alpha}}\left[\frac{\alpha}{a}+\frac{a}{\alpha}\right]$. Although the dependence upon $\alpha$ obviously restricts the predictive powers of our analysis, it does not however prevent us from drawing a schematic phase diagram: for this purpose it is acceptable to choose $\alpha=$ constant. Without loss of generality we therefore set $k$ equal to unity, being equivalent to renormalizing the Coulomb interaction $G \rightarrow G / \sqrt{k}$. In the QFKM's phase diagram presented in Fig. (11) we plot the ratio $G^{2} / t V$ as a function of $n_{0}^{c}$. We note that the phase diagram for $0.5<n_{0}^{c} \leq 1$ can be obtained by reflecting Fig. (II) along the line $n_{0}^{c}=0.5$. The only significant difference to Fig. (III) is that the SEG phase is replaced by the "empty" state $\left(n^{f}=0\right)$. We obtain the $V \rightarrow 0$ weak-coupling crystalline phases in the phase diagram on the inclusion of the backscattering corrections in Eq. (5). Physically, since a finite hybridization is always 
present, a MV state will be realized instead of these phases, as we have observed above.

Starting in the segregated phase $(\delta \ll 0)$ we lower $G$. Below the value $G=\sqrt{\pi / 2} G_{c}$, the Ising coupling in Eq. (6) is no longer greater than the transverse field everywhere on the lattice. The rare regions where the transverse field term is strongest breaks the single $f$-electron block up into randomly distributed large clusters separated by regions of the MV phase: this local competition between the MV and SEG phases may be regarded as a charge analogy of the Griffiths phase [G1 in Fig. (10] .21 $^{21}$ Although such a state has been inferred by numerical work, this is the first analytic indication of its existence ${ }^{22}$ The system still, however, retains recognizable features of the SEG phase: the mean $f-f$ correlations $\overline{\left\langle n_{j}^{f} n_{j+x}^{f}\right\rangle}$ decay exponentially towards $\left(n^{f}\right)^{2}>\left(n_{0}^{f}\right)^{2}$ with a correlation length $\xi \sim e^{-2 \delta^{2}}$, the non-universal power-law dependence reflecting the continued presence of the infinite $f$-cluster throughout G1.

As $G$ is lowered the Griffiths phase G1 gives way to the quantum critical regime (QCR). The QCR is dominated by the physics of the critical point: at every length scale we find the coexistence of equal regions of mixed- and integer-valence. Due to the $f$-level renormalization we do not find algebraic decay of correlation functions, but rather exponential attenuation with a correlation length of $\xi \sim 1+\mathcal{O}\left(\delta^{2}\right)$. For $n_{0}^{c}=0.5$ the $f$-level shifting vanishes and so the QCR shrinks down to a single quantum critical point (QCP) characterized by dynamical critical exponent $z=\infty$ at $G=G_{c}\left(n_{0}^{c}=0.5\right)=\sqrt{t V}$. Note that the precise position of the QCP is dependent upon the choice of $\alpha !^{17}$

Further decreasing $G$ below the QCR, we reach a second Griffiths phase (G2) with opposite character to G1: here the hybridization term dominates the segregating interaction over most of the lattice. The SEG phase almost completely disappears, with only rare clusters of valence-ordered localized orbitals embedded in an MV background remaining of this state. The mean correlation functions decay exponentially, although less rapidly than in the G1, with $\xi \sim\left(1+\delta^{-2}\right)^{-1}$. Since the minimum magnitude that the transverse field in $\mathcal{H}_{\text {eff }}$ assumes is $\min \left|\widetilde{h}_{j}^{x}\right|=0$, the weakly disordered phase should be present for any $G>0$; for $\delta \gg 1$ the integer-valence clusters are however extremely rare and the last two terms of $\mathcal{H}_{\text {eff }}$ dictate the physics. As such, we can justifiably identify the very low- $G$ behaviour as typical of a MV state [Fig. (11)].

The characteristic feature of the Griffiths phases and the QCR is the local competition between the MV and SEG states. This manifests itself as the co-existing clusters of 
$f$-orbitals with mixed- and integer-valence. The integer-valence clusters are of greatest interest: these correspond to the dressing of a $c$-electron by a "cloud" of near-empty $f$-orbitals. This coupling of the $c$ - and $f$-electron densities originates from the forward-scattering, and constitutes an electronic polaron. Electronic polarons form only at intermediate-coupling: at weak-coupling the screening clouds are heavily suppressed by the resonant (MV) scattering with the $f$-orbitals whereas at strong coupling the clouds merge to form the SEG phase. Since the bosonization method treats the forward-scattering exactly, our discovery of electronic polarons within a crossover regime between mixed- and integer-valence phases rigorously confirms the scenario proposed by Liu and Ho $\stackrel{?}{\underline{T}}^{-}$

Note that in the Griffiths phases which flank the sides of the QCR the length scale $\xi$ differs from the conduction electron mean free path which gives rise to competing time scales: slow motion of the electronic polarons and fast motion of the conduction electrons. The different dynamics of the two types of particles provides a close analogy to a two fluid scenario. Since the polarons are randomly distributed across the lattice, these states can be viewed as intrinsic inhomogeneities involving charge fluctuations and short-range charge correlations. This resembles very closely the spin polaron liquid found in the 1D Kondo lattice model. $\stackrel{23}{ }$

Summarizing our results, we have derived an effective model for the occupation of the localized orbitals in the 1D QFKM. This model predicts a crossover from a MV state at weak $c-f$ Coulomb repulsion $G$ to the integer-valence SEG state with increasing coupling strength. At intermediate values of the coupling the system exhibits an electronic Griffiths phase. The effective Hamiltonian gives a detailed accounting of the $f$-electron physics across the phase diagram, confirming the importance of electronic polarons in the QFKM. An extensive discussion of this system is to follow in a later paper ${ }^{24}$.

1 L. M. Falicov and J. C. Kimball, Phys. Rev. Lett. 22, 997 (1969).

2 J. M. Lawrence, P. S. Riseborough and R. D. Parks, Rep. Prog. Phys. 44, 1 (1981).

3 H. J. Leder, Solid State Comm. 27, 579 (1979); W. Hanke and J. E. Hirsch, Phys. Rev. B 25, $6748(1982)$.

4 T. Portengen, Th. Östreich and L. J. Sham, Phys. Rev. B 54, 17452 (1996). 
5 P. Schlottmann, Solid State Comm. 31, 885 (1979); Phys. Rev. B 22, 622 (1980).

6 P. Schlottmann, Phys. Rev. B 22, 613 (1980).

7 S. H. Liu and K.-M. Ho, Phys. Rev. B 30, 3039 (1984); S. H. Liu, Phys. Rev. Lett. 58, 2706 (1987).

8 Y. Toyozawa, Prog. Theor. Phys. 12, 421 (1954).

9 A. Ślebarski et al., Phys. Rev. B 69, 155118 (2004); N. E. Sluchanko et al., cond-mat/0505386 (unpublished).

10 Y. Onishi and K. Miyake, J. Phys. Soc. Jpn. 69, 3955 (2000) ; A. T. Holmes, D. Jaccard and K. Miyake, Phys. Rev. B 69, 024508 (2004).

11 M. Gulácsi, Adv. Phys. 53, 769 (2004).

12 J. von Delft and H. Schoeller, Ann. der Physik 4, 223 (1998).

13 K. D. Schotte and U. Schotte, Phys. Rev. 182, 479 (1969).

14 P. Farkašovský, Intl. J. Mod. Phys. B 17, 4897 (2003).

15 P. Lemberger, J. Phys. A: Math. Gen. 25, 715 (1992).

16 J. K. Freericks and L. M. Falicov, Phys. Rev. B 41, 2163 (1990).

17 P. M. R. Brydon and M. Gulácsi, cond-mat/0506218 (unpublished).

18 K. Uzelac, R. Jullien and P. Pfeuty, Phys. Rev. B 22, 436 (1980).

19 I. I. Satija and M. M. Doria, Phys. Rev. B 39, 9757 (1989); I. I. Satija, Phys. Rev. B 41, 7235 (1990).

20 S. Jitormirskaya and A. Klein, J. Stat. Phys. 73, 319 (1993).

21 D. S. Fisher, Phys. Rev. B 51, 6411 (1995).

22 E. Miranda and V. Dobrosavljević, Phys. Rev. Lett. 86, 264 (2001).

23 I. P. McCulloch, A. Juozapavicius, A. Rosengren and M. Gulácsi, Phys. Rev. B 65, 052410 (2002).

24 P. M. R. Brydon and M. Gulácsi, in preparation. 


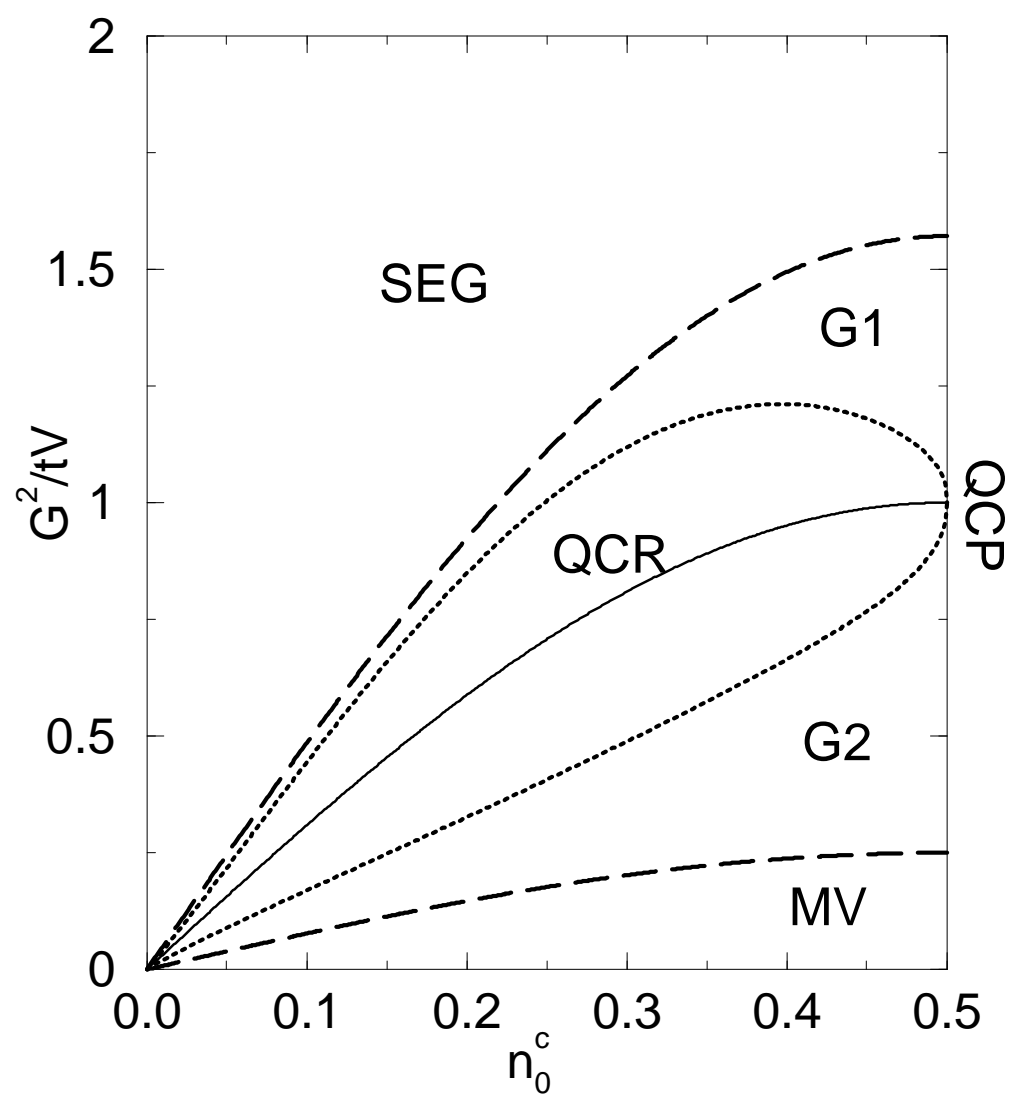

FIG. 1: Ground-state phase diagram for the QFKM below half-filling. The quantum critical regime (QCR) is found on either side of the solid line $\delta=0$. Along the line $n_{0}^{c}=0.5$ the QCR is replaced by a quantum critical point (QCP). The dotted lines denote the approximate "boundary" of the QCR with the Griffiths phases G1 and G2. The dashed lines separate these Griffiths phases from the segregated (SEG) and mixed-valence (MV) phases. Electronic polarons are present in both Griffiths phases and the QCR. 